\title{
IMAGE QUALITY ASSESSING BY USING NN AND SVM
}

\author{
YU-BING TONG, QING CHANG, QI-SHAN ZHANG \\ School of Electronics and Information Engineering, Beihang University, Beijing 100083, China \\ E-MAIL: ybtong@ee.buaa.edu.cn, changq@263.net, zhangqishan@263.net
}

\begin{abstract}
:
In the correlative curve of image subjective and objective quality assessing, there are some points that lower the performance of image quality assessing model. In this paper, the concept of isolated points was given and isolated points predicting was also illuminated. A new model was given based on NN-Neural Network and SVM-Support Vector Machines with PSNR and SSIM-Structure Similarity, which were used as two indexes describing image quality. NN was used to obtain the mapping functions between objective quality assessing indexes and subjective quality assessing value. SVM was used to classify the images into different types. Then the images were accessed by using different mapping functions. The number of isolated points was reduced in the correlative curve of the new model. The results from simulation experiment showed the model was effective. The monotony of the model is $6.94 \%$ higher than PSNR and RMSE-root mean square error is $35.90 \%$ higher than PSNR.
\end{abstract}

\section{Keywords:}

Neural network; Support vector machines; Image quality assessing; PSNR

\section{Introduction}

Recently, more attention has been placed on objective quality assessing models that are expected to reflect images subjective quality exactly. The main task is to reduce the offset between subjective assessing and objective assessing. Most images are assessed based on HVS-Human Visual System. HVS is more complicated than PSNR and some physiological and psychophysical characteristics of HVS are not clear so far. PSNR does not involve much characteristic information of image, so the visible offset maybe generated between PSNR and subjective quality. SSIM is the method used to assess structure similarity between the original signal and the reconstructed signal ${ }^{[1]}$. The information of image structure is used in SSIM. In this paper, PSNR and SSIM were used as two indexes describing image quality. A new model was given here based on NN-Neural Network and SVM-Support Vector Machines. The results from our experiment by using
UTexas's database showed the model output could effectively reflect the image subjective quality ${ }^{[2]}$.

In Section II, image quality assessing by using PSNR and SSIM were analyzed. The new model by using NN and SVM was described in Section III. Simulation experiment and result analysis were made in Section IV.And finally, conclusions were made in Section $\mathrm{V}$.

\section{Analysis of PSNR and SSIM}

PSNR is defined as follows:

$$
P S N R=10 \lg \left(\frac{255^{2} \cdot M \cdot N}{\sum_{i=1}^{M} \sum_{j=1}^{N}[a(i, j)-\hat{a}(i, j)]^{2}}\right)
$$

$\hat{a}_{1}(i, j)$ and $\hat{a}_{2}(i, j)$ are two distorted images with the same PSNR, for the simplest case,

if

$$
\left|a(i, j)-a_{1}(\hat{i, j})\right|=\left|a(i, j)-a_{2}(\hat{i}, j)\right|,
$$

then

$\hat{a}_{1}(i, j)=\hat{a}_{2}(i, j)$ or $\hat{a}_{1}(i, j)+\hat{a}_{2}(i, j)=2 a(i, j)$

So $\hat{a}_{1}(i, j)$ and $\hat{a}_{2}(i, j)$ may be not equal with each other or even looks much different. PSNR is the common index for assessing signal quality and is also valid for most images. If bad cases generated by assessing with PSNR were reduced, PSNR would be more efficient for image quality assessing. In this paper, how to reduce the worse case was mainly concerned.

With the assumption that HVS is highly adapted to extract structural information from the viewing field, SSIM provide a good approximation to image distortion by measuring structural information change. SSIM is defined as follows: 


$$
\operatorname{SSIM}(x, y)=[l(x, y)]^{\alpha} \cdot[c(x, y)]^{\beta} \cdot[s(x, y)]^{\gamma}
$$

$l(x, y), c(x, y)$ and $s(x, y)$ are luminance, contrast and structure comparing functions, $\alpha, \beta, \gamma>0$. But in fact, the mapping function among $\operatorname{SSIM}(\mathrm{x}, \mathrm{y})$ and $l(x, y), c(x, y), s(x, y)$ should be nonlinear and probably different with equation(2). There are still some points whose objective assessing value is far away from subjective value in the curve of subjective-objective quality assessing with SSIM. Those points lower the performance of image quality assessing model. If the assessing value of these points were modified and there were less number of such points, the model output would be more closely related with the subjective quality assessing. SSIM and PSNR were used together here to set up new model..

\section{New image quality assessing model based on $\mathrm{NN}$ and} SVM

NN and SVM were combined to set up new model with two image quality assessing indexes, PSNR and SSIM. The flow was shown as follows

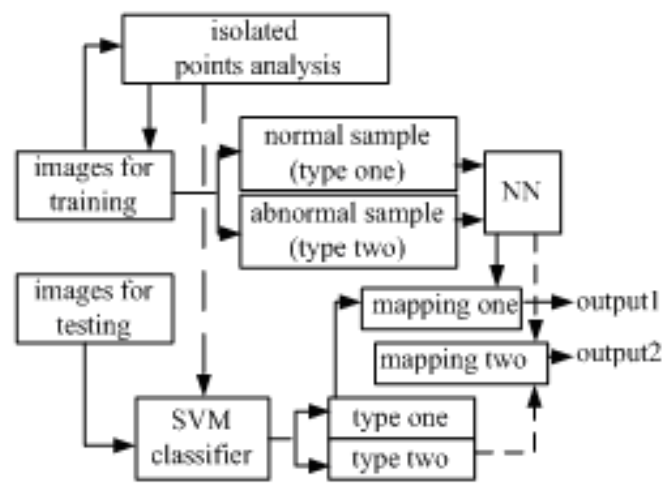

Figure.1 the flow of image quality assessing

Images quality assessing was divided into two parts. One was the part for training data; the other was for testing data. In the first part, isolated points analysis was made and the mapping functions between objective quality assessing and subjective quality assessing was obtained by using NN. In the second part, isolated points predicting was made and the testing images was assessed by using the new model.

(1) Isolated points analysis and mapping functions for image quality assessing
Definition 1: In the correlative curve of subjective quality assessing and objective assessing, if the offset between them is larger than a threshold, $T H$, then the corresponding image to that point could be defined as isolated point. $1^{\text {st }}$ way(space istance): $\left|M O S_{\text {subjective }}-E V A_{\text {objective }}\right| \geq T H$ $2^{\text {nd }}$ way(slope ratio): $\left\{\begin{array}{l}\left(\operatorname{MOS}_{\text {subjective }} / E V A_{\text {objective }}\right) \geq T H \\ \text { or } \\ \left(M O S_{\text {subjective }} / E V A_{\text {objective }}\right) \leq 1 / T H\end{array}\right.$

Where, $T H$, the threshold that could be derived from experiments, $M O S_{\text {subjective }}$, the subjective quality assessing value, $E V A_{\text {objective }}$, the objective quality assessing value. In this section, the offset in $2^{\text {nd }}$ way was used and the correlative curve of subjective quality assessing and objective assessing is shown in Figure.2. The isolated points were denoted with "+".

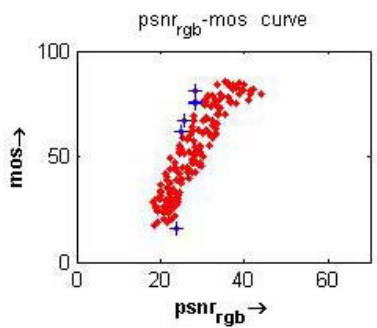

(a) PSNR-MOS

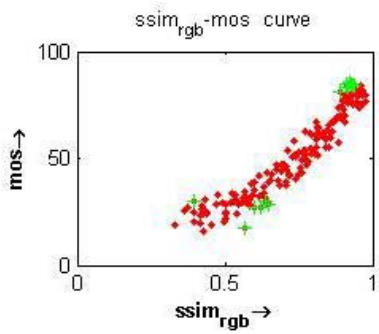

(b) SSIM-MOS
Figure. 2 The associated curve of subjective quality assessing and objective assessing

From the above definition, the training data set was divided into two subsets, normal set and abnormal set. Then the subsets were respectively used to train BP neural network and finally two mappings were obtained. Here, the isolated points included those both in PSNR-MOS and SSIM-MOS. And the number of isolated points could also be controlled by adjusting the threshold in definition 1. Different kinds of images would be assessed by using different mappings with the following structure in Figure.3.

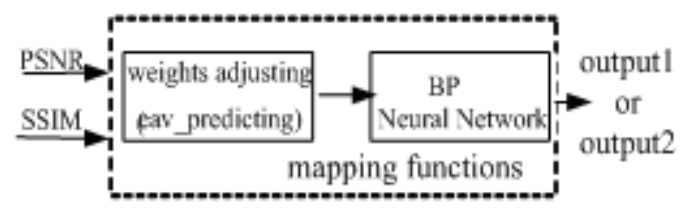

Figure.3 mappings for different kinds of image quality assessing 
PSNR and SSIM were added with weights before using BP neural networking,

$$
\text { eav_predicting }=p \cdot P S N R+s \cdot S S I M
$$

$p, s$ are the weights, which were derived from experiments.

For normal samples, $p=0.4, s=0.6$. For abnormal samples, $p=0.6, s=0.4$. Using training data, BP-NN could obtain final mapping functions through adjusting the weights among network nodes.

(2) isolated points predicting with SVM classifier Isolated points were defined using subjective quality assessing $M O S_{\text {subjective }}$, but in fact there are often no subjective assessing when assessing image quality. Here, isolated point predicting was made by using SVM classifier, which classified samples into two kinds without using $M O S_{\text {subjective }}$.

SVM uses kernel function to map the data in input space to high-dimensional features space where the data becomes linearly separable. SVM need obtain the generalized optimal classifying plane with the following form,

$$
\begin{aligned}
y & =f(x ; a) \\
& =\sum_{i=1}^{N}\left(a_{i}^{*}-a_{i}\right)\left(x_{i} \times x\right)+b \\
& =\sum_{i=1}^{N}\left(a_{i}^{*}-a_{i}\right) K\left(x, x_{i}\right)+b
\end{aligned}
$$

$K(\cdot)=,\left(x_{i} \cdot x\right)$, kernel function satisfying Mercey theory; $b$, a constant; $a, a^{*}$, the optimal results of a Quadratic Programming (QP) problem ${ }^{[3]}$. Here, SVM classifier was set using the results of isolated points analyzing. Then image to be assessed was classified into different kinds with different value. One means normal assessing of image quality. Negative one means abnormal assessing of image quality, which means isolated points would be generated. The distance between the different kinds would be largest based on SVM classifier.

\section{Experiment and results analysis}

The correlative curve of subjective quality assessing and objective assessing by using th e new model is in Figure.4.

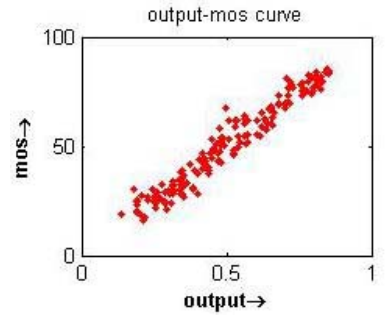

(a) using training data

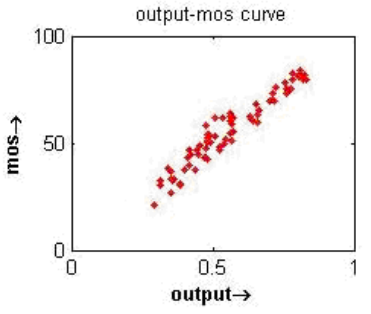

(b) using testing data
Figure. 4 The associated curve of subjective quality assessing and objective assessing

The number of isolated points is lower in the output-mos curve than that in PSNR-MOS curve or SSIM-MOS curve. According to VQEG's final report and test paln ${ }^{[4,5]}$, Table 1 list the statistic data used to evaluate the performance of image quality assessing model with two way of describing isolated points. RMSE is root mean square error; $r_{p}$ is the person linear correlation coefficient between objective assessing value and MOS. Then $r_{p}$ provides an evaluation of prediction monotony. The outlier ratio gives the evaluation of prediction consistency of a model. With the same level of prediction consistency, the monotony of the model is $7.42 \%$ higher than PSNR, the rmse is $36.06 \%$ higher than PSNR.

Table 1 statistic data for the image quality assessing

\begin{tabular}{|c|c|c|c|c|c|c|}
\hline \multirow{2}{*}{} & \multicolumn{2}{|c|}{$\mathrm{Rp}$} & \multicolumn{2}{c|}{ outlier } & \multicolumn{2}{c|}{ rmse } \\
\cline { 2 - 7 } & $\begin{array}{c}\text { space } \\
\text { distance }\end{array}$ & $\begin{array}{c}\text { slope } \\
\text { ratio }\end{array}$ & $\begin{array}{c}\text { space } \\
\text { distance }\end{array}$ & $\begin{array}{c}\text { slope } \\
\text { ratio }\end{array}$ & $\begin{array}{c}\text { space } \\
\text { distance }\end{array}$ & $\begin{array}{c}\text { slope } \\
\text { ratio }\end{array}$ \\
\hline PSNR & \multicolumn{2}{|c|}{0.8979} & \multicolumn{2}{|c|}{0} & \multicolumn{2}{c|}{0.3053} \\
\hline SSIM & \multicolumn{2}{|c|}{0.9601} & \multicolumn{2}{|c|}{0.3947} & \multicolumn{2}{c|}{0.4860} \\
\hline new_model(output) & 0.9602 & 0.9645 & 0 & 0 & 0.1957 & 0.1952 \\
\hline $\begin{array}{c}\text { Contrasted with } \\
\text { PSNR }\end{array}$ & $6.94 \%$ & $7.42 \%$ & & & $35.90 \%$ & $36.06 \%$ \\
\hline
\end{tabular}

For Figure.5 and Figure.6, the objective assessing value of the distorted image derived from our new model was 0.63 with PSNR being $28.7 \mathrm{db}$ and SSIM being 0.98 . The highest score was 1 without any loss between two images. The quality of Figure. 6 was much worse than that of Figure.5. But Figure. 6 could also be distinguishable. So the results of the new model would be more close to the subjective quality assessing. 

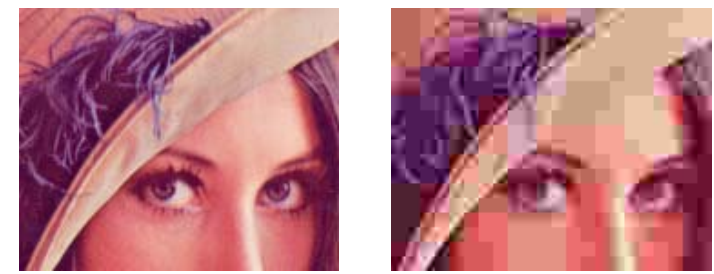

Figure. 5 the original image Figure. 6 the distorted image

\section{Conclusions}

In this paper, the definition of isolated points was given. Based on the isolated points analysis, Neural network and SVM classifier were used to set up new image quality assessing model with PSNR and SSIM as two images quality indexes. The experiment results show that the proposed model can reflect image quality effectively. The number of isolated points in the performance curve was reduced. To get more accurate assessing, more characteristics of HVS should also be researched to find new high-performance quality assessing models.

\section{References}

[1] Zhou Wang, Liang Lu, Alan C.Bovik, Video Quality Assessment Using Structural Distortion Measurement, IEEE ICIP 2002, III-65-68.

[2] http://live.ece.utexas.edu/index.htm, 2005.1.10.

[3] Vladimir N. Vapnik, An overview of statistical learning theory $[\mathrm{J}]$, Neural Networks, IEEE Transactions on, Volume: 10, Issue: 5, Sept. 1999. Pages: 988 - 999.

[4] http://www.vqeg.org, Final Report From the Video Quality Experts Group on the Validation of Objective Models of Video Quality Assessment,2005.1.10[EB/OL].

[5] http://www.vqeg.org, VQEG RRNR-TV Group TEST PLAN, Draft version 1.7, 2004.6.21[EB/OL], 2005.1.10. 